\title{
Infusão Calórica e Proteica versus Prescrição Dietética na Terapia Nutricional Enteral do Paciente Oncológico
}

doi: https://doi.org/10.32635/2176-9745.RBC.2021v67n3.1275

Caloric and Protein Infusion versus Dietary Prescription in Enteral Nutritional Therapy for Cancer Patients
Infusión de Calorías y Proteínas versus Prescripción Dietética en la Terapia Nutricional Enteral para Pacientes con Cáncer

Mikelly Joanny da Silva'; Thalia de Jesus Souza da Silva²; Jorge Antonio Pavão Cordeiro Sobrinho ${ }^{3}$; Erika Ribeiro Garcia ${ }^{4}$; Maria Tereza Silva de Medeiros ${ }^{5}$; Thalita Albuquerque Véras Câmara ${ }^{6}$; Rosângela Maria Lopes de Sousa ; Alexsandro Ferreira dos Santos ${ }^{8}$

\section{RESUMO}

Introduçáo: $\mathrm{O}$ suporte nutricional no paciente oncológico não se restringe ao cálculo das necessidades nutricionais e à prescrição dietética, é necessário que o volume prescrito seja realmente infundido. Objetivo: Comparar a infusão calórica e proteica com a prescrição dietética em pacientes oncológicos sob terapia nutricional enteral. Método: Estudo retrospectivo, analítico, conduzido em fichas de acompanhamento nutricional de pacientes que estiveram internados durante sete dias no mínimo, exclusivamente sob terapia nutricional enteral por sonda ou ostomia entre janeiro/2009 e dezembro/2012. As fichas que atenderam aos critérios de seleção foram ordenadas alfabeticamente e, aleatoriamente, selecionadas por intervalo de retirada $(\mathrm{k}=5)$. Coletaram-se: idade, sexo, localização tumoral, composição nutricional, volume prescrito e aquele infundido da fórmula enteral utilizada. Com estes dois últimos, calculou-se o indicador "prescrição versus infusão". Os testes t Student, t pareado e qui-quadrado foram aplicados a um nível de significância máximo de 5\%. Resultados: Das 120 fichas analisadas, prevaleceram homens $(61,7 \%)$ com idade média de 58,3 16,8anos. Em 2010, houve maiores médias de diferença entre volume prescrito e infundido $(-392,64 \mathrm{ml})$ e calorias prescritas versus infundidas $(-528,23 \mathrm{cal})$. Apenas $37,5 \%$ dos pacientes receberam um mínimo de $70 \%$ do volume prescrito. Conclusáo: Houve um inadequado consumo da dieta infundida em relação à prescrita. Sugere-se que intervençóes pela equipe multiprofissional em terapia nutricional minimizem as intercorrências encontradas.

Palavras-chave: Terapia Nutricional; Nutrição Enteral; Neoplasias.

\section{ABSTRACT}

Introduction: Nutritional support for cancer patients is not restricted to the calculation of nutritional needs and dietary prescription, it is necessary that the prescribed volume is actually infused. Objective: To compare caloric and protein infusion with dietary prescription in cancer patients undergoing enteral nutritional therapy. Method: Retrospective, analytical study, conducted on nutritional monitoring cards who were hospitalized at least for seven days, exclusively under enteral nutritional therapy by tube or ostomy, between January 2009 and December 2012. The cards that met the selection criteria were ordered alphabetically and randomly selected by withdrawal interval $(\mathrm{k}=5)$. It was collected: age, sex, tumor location, nutritional composition, prescribed and infused volume of the enteral formula utilized. With these last two, the indicator "prescription versus infusion" was calculated. Student $t$ test, paired t test and chi-square test were applied at a maximum significance level of 5\%. Results: Of the 120 cards analyzed, men $(61.7 \%)$ were more prevalent with mean age of $58.3 \pm 16.8$ years. In 2010, higher means of the difference between prescribed and infused volume $(-392.64 \mathrm{ml})$ and prescribed versus infused calories $(-528.23$ cal) were observed. Only $37.5 \%$ of the patients received a minimum of $70 \%$ of the prescribed volume. Conclusion: There was an inadequate intake of the infused in relation to the prescribed diet. It is suggested nutritional therapy interventions from the multi-professional team to minimize the complications encountered.

Key words: Nutrition Therapy; Enteral Nutrition; Neoplasms.

\section{RESUMEN}

Introducción: El soporte nutricional en pacientes oncológicos no se limita al cálculo de necesidades nutricionales y prescripción dietética, es necesario que el volumen prescrito sea efectivamente infundido. Objetivo: Comparar la infusión calórica y proteica con la prescripción dietética en pacientes oncológicos sometidos a terapia nutricional enteral. Método: Estudio retrospectivo, analítico, realizado en formularios de seguimiento de pacientes que estuvieron hospitalizados durante al menos siete días, exclusivamente bajo terapia nutricional enteral por sonda u ostomía entre enero/2009 a diciembre/2012. Los formularios que cumplieron con los criterios de selección se ordenaron alfabéticamente y se seleccionaron aleatoriamente por intervalo de retiro $(k=5)$. Se recogió: edad, sexo, localización del tumor, composición nutricional, volumen prescrito y el infundido con la fórmula enteral empleada. Con estos dos últimos se calculó el indicador "receta versus infusión". La prueba t de Student, la prueba t pareada y la prueba de chi-cuadrado se aplicaron a un nivel máximo de significancia del 5\%. Resultados: De los 120 registros analizados, predominó: hombres $(61,7 \%)$, con una edad promedio de 58,3 $\pm 16,8$ años. En 2010, hubo mayores promedios de diferencia entre el volumen prescrito y el infundido $(-392,64 \mathrm{ml})$ y las calorías prescritas frente a las infundidas $(-528,23 \mathrm{cal})$. Solo el $37,5 \%$ de los pacientes recibió un mínimo del 70\% del volumen prescrito. Conclusión: Existió un consumo inadecuado de la dieta infundida en relación con la prescrita. Se sugiere que intervenciones del equipo multiprofesional en terapia nutricional minimicen las complicaciones encontradas.

Palabras clave: Terapia Nutricional; Nutrición Enteral; Neoplasias.

\footnotetext{
1,2,3,5,6,8 Curso de Nutrição da Faculdade Santa Terezinha (CEST). São Luís (MA), Brasil.

4,7Universidade Ceuma (Uniceuma). São Luís (MA), Brasil.

'E-mail: mikellyjoanny@hotmail.com. Orcid iD: https://orcid.org/0000-0002-2625-2615

${ }^{2}$ E-mail: thaliasilva@hotmail.com. Orcid iD: https://orcid.org/0000-0003-4766-9847

${ }^{3}$ E-mail: jorgepavaopp@hotmail.com. Orcid iD: https://orcid.org/0000-0003-1478-0039

${ }^{4}$ E-mail: erikka.garcya@gmail.com. Orcid iD: https://orcid.org/0000-0002-6005-8883

${ }^{5}$ E-mail: m.mdm@uol.com.br. Orcid iD: https://orcid.org/0000-0002-1653-8804

${ }^{6}$ E-mail: thalitacamara3101@hotmail.com. Orcid iD: https://orcid.org/0000-0003-1711-8331

7E-mail: rmls33@hotmail.com. Orcid iD: https://orcid.org/0000-0003-4728-9318

${ }^{8}$ E-mail: fs_alexsandro@yahoo.com.br. Orcid iD: https://orcid.org/0000-0001-7470-4607

Endereço para correspondência: Erika Ribeiro Garcia.Travessa Bom Jesus. Casa 4 - Anjo da Guarda. São Luís (MA), Brasil. CEP 65085-070. E-mail: erikka.garcya@gmail.com.
} 


\section{INTRODUÇÃO}

O câncer tornou-se um problema de saúde pública no mundo e no Brasil ${ }^{1}$. É uma doença catabólica que atua consumindo reservas nutricionais do paciente, levando a um prejuízo nutricional ${ }^{2}$.

Os casos de câncer em sua maioria são diagnosticados tardiamente, quando os pacientes já apresentam desnutrição, e parte desses pacientes desenvolve caquexia, o que eleva as chances de morte ${ }^{3}$.

A equipe multiprofissional de terapia nutricional, juntamente com o profissional nutricionista, torna-se indispensável para estímulo de um aporte nutricional adequado, frente aos efeitos colaterais apresentados no tratamento oncológico e à vigência de fatores pró-caquéticos, que, combinados, podem levar à perda de peso e à diminuição da imunidade ${ }^{4}$.

A terapia nutricional enteral (TNE) tem demonstrado inúmeras vantagens para os pacientes hospitalizados, surgindo como recurso terapêutico de manutenção e recuperação do estado nutricional de pacientes com câncer, trazendo como benefícios: redução do custo do tratamento e menor tempo de internação, melhora na resposta imunológica e das condiçôes clínicas 5 .

As alteraçóes metabólicas no câncer contribuem para o aumento do gasto energético e podem resultar em perdas progressivas. Somado a isto, os pacientes com câncer podem apresentar: anorexia, depleção da musculatura esquelética e perda tecidual, colaborando ainda mais para o processo de desnutrição ${ }^{6}$.

Paralelo a isto, o tratamento induz os pacientes com câncer a diversas alteraçóes nutricionais em razão dos seus efeitos colaterais ${ }^{7}$.

O suporte nutricional do paciente com câncer não se limita somente ao cálculo das necessidades nutricionais e à sua prescriçãao dietética. Pois, tão importante quanto à prescrição da terapia nutricional adequada, é a certeza de que ele realmente receberá o volume prescrito. A atençáo nutricional e o conhecimento dos fatores que interferem na efetiva administração da TNE permitem a adoçấo de medidas com aporte calórico proteico adequado aos pacientes com câncer. Por esse motivo, a introdução precoce e adequada da TNE pode reduzir consideravelmente a incidência de infecçóes, o aparecimento de úlceras por pressão e o tempo de permanência hospitalar, entre outros fatores ${ }^{8}$.

A administração da TNE é quase sempre prejudicada e cerca de $29 \%$ dos pacientes em nutrição enteral não recebem o que lhes é prescrito diariamente?. Os fatores corriqueiramente envolvidos são: instabilidade hemodinâmica, jejum para exames e procedimentos médicos, de enfermagem e de fisioterapia, recusa do paciente, obstruçấo da sonda, entre outros ${ }^{9}$.
Durante a TNE, é importante que seja feita a verificação diária das intercorrências para que seja possível analisar o aproveitamento total da dieta e das complicaçóes que podem ser controladas como a manipulaçáo, a composição nutricional, o tempo de administração da fórmula e o fornecimento de calorias de acordo com a condição clínica do paciente ${ }^{10}$.

Ultimamente, diversos estudos procuraram analisar as não conformidades entre a quantidade de calorias e proteínas prescritas com a de fato administrada, além dos fatores que contribuem para a interrupção da TNE. Pesquisas apontam valores de inadequação entre 50\% e $90 \%$ do percentual administrado em relação às reais necessidades nutricionais dos pacientes. Contudo, nenhuma direcionou-se a pacientes com câncer ${ }^{11-13}$.

Neste contexto, o indicador de qualidade em "terapia nutricional volume infundido versus volume prescrito" pode garantir a recuperação ou a manutençáo do estado nutricional ${ }^{11}$.

Assim, o objetivo do estudo é estimar e analisar a infusão versus a prescrição de volume de TNE em pacientes oncológicos, bem como estabelecer a obediência ao indicador de qualidade em "terapia nutricional volume infundido versus volume prescrito".

\section{MÉTODO}

Foi conduzido um estudo observacional, retrospectivo, analítico, com coleta de dados secundária.

Esta pesquisa foi autorizada pelo Hospital do Câncer Aldenora Bello, localizado na cidade de São Luís-MA e aprovado pelo Comitê de Ética em pesquisa da Universidade Ceuma (Uniceuma), sob o parecer número 575.933/ 2014.

Para a coleta de dados, foram pesquisadas, no Serviço de Nutrição e Dietética do hospital, as fichas de acompanhamento de nutrição enteral de pacientes que já haviam recebido alta hospitalar e que estiveram internados, exclusivamente sob o uso de TNE por sonda ou ostomia, no período de janeiro de 2009 a dezembro de 2012.

Foram coletadas 1.190 fichas de pacientes de ambos os sexos. Selecionaram-se, a partir dos critérios de inclusão, adultos (acima de 19 anos e máximo de 59 anos) e idosos (mínimo de 60 anos), que estiveram sob TNE exclusiva por sonda ou ostomia, por sete dias ou mais de acompanhamento, e atendidos no mesmo período.

Foi realizada a aplicação do indicador de qualidade em "terapia nutricional volume infundido versus volume prescrito", que possui como objetivo estratégico conhecer o volume de nutriçáo enteral infundido versus o volume prescrito, esse indicador possui como meta que pelo menos $80 \%$ dos pacientes sob TNE por sonda recebam 
um mínimo de $70 \%$ do volume que lhes foi prescrito ${ }^{14}$.

Oitocentas fichas referentes aos pacientes submetidos à TNE inferior a sete dias ou fora do período estabelecido para coleta não foram incluídas. Como critérios de exclusão, destacaram-se ainda as fichas de crianças e adolescentes e de pacientes que estiveram sob terapia nutricional mista $(\mathrm{n}=270)$.

As fichas que atenderam aos critérios de inclusão foram organizadas por ordem alfabética; posteriormente, selecionaram-se 140 fichas de forma aleatória e não probabilística, por meio de intervalo de retirada $(\mathrm{k}=5)$, para assim dar-se início à coleta das informaçóes. Das 140 fichas escolhidas, 20 foram excluídas por inconsistência nos dados, resultando em uma amostra de 120 .

Das fichas elegíveis, as variáveis analisadas foram a composição nutricional (densidade calórica e quantidade de proteínas, carboidratos e lipídios), os volumes prescritos e infundidos das fórmulas enterais - com estes, calculou-se o aporte nutricional prescrito - e o efetivamente infundido, além da localização do tumor, idade, sexo e causa das intercorrências da TNE.

Foram avaliados quatro dias, sendo o primeiro dia de acompanhamento nutricional (D1) e D4 o último de dia, os D2 e D3 foram de acompanhamento da TNE, intermediários entre D1 e D4, escolhidos a partir de sorteio.

Os dados foram expressos em frequências (absoluta e relativa) em variáveis categóricas, e média e desvio-padrão em variáveis contínuas. Sendo o banco de dados formulado em Microsoft Excel $^{\bullet}$ (2010).

Para verificar a relação entre o percentual de infusão e variáveis categóricas, foi aplicado o teste do qui-quadrado $\left(\mathrm{x}^{2}\right)$.

Para a comparação da média da idade em relação à meta de infusão, foi aplicado o teste t de Student. Já para averiguar diferenças entre os volumes de enteral infundido e prescrito, e ainda a diferença do volume calórico infundido e prescrito, foi aplicado o teste t pareado.

Utilizou-se a análise de variância ANOVA para comparação entre os volumes e aportes calóricos prescritos e os efetivamente infundidos ao longo dos anos.

A normalidade foi comprovada a partir do teste Shapiro Wilk. O programa estatístico utilizado foi o Stata ${ }^{\oplus}$ (versão 12). Para a interpretação estatísticas dos resultados, em todos os testes foi adotado nível de significância alfa inferior a 0,05 .

\section{RESULTADOS}

Das 120 fichas analisadas, prevaleceram homens $(61,7 \%)$, com localização tumoral de cabeça e pescoço (48,3\%), seguida pelo trato gastrointestinal $(22,5 \%)$. A média de idade foi de $58,3 \pm 16,8$ anos e a faixa etária mais frequente a de 38 a 54 anos (38,3\%), conforme a Tabela 1 .

Notou-se, inicialmente (D1), que a infusão de TNE

Tabela 1. Caracterização amostral de pacientes oncológicos em terapia enteral. São Luís, Maranhão, Brasil

\begin{tabular}{lcc}
\hline \multicolumn{1}{c}{ Variáveis } & $\mathrm{n}$ & $\%$ \\
\hline Sexo & & \\
Feminino & 46 & 38,3 \\
Masculino & 74 & 61,7 \\
Localização do tumor & & \\
Cabeça e pescoço & 58 & 48,3 \\
Trato gastrointestinal & 27 & 22,5 \\
Ginecológico e mama & 16 & 13,3 \\
Aparelho reprodutor masculino & 11 & 9,2 \\
Outros & 8 & 6,63 \\
Idade (anos) & & \\
$21-37$ & 2 & 1,7 \\
$38-54$ & 46 & 38,3 \\
$55-70$ & 42 & 35,0 \\
$71-87$ & 30 & 25,0 \\
\hline Total & 120 & 100,0 \\
\hline
\end{tabular}

é reduzida, tanto para o volume prescrito $(957,9 \pm 392,6$ $\mathrm{ml})$, quanto para o volume infundido $(480,9 \pm 244,9 \mathrm{ml})$. A infusão alcança picos máximos no seguimento da terapia nutricional (D2 e D3) e reduz novamente ao término (D4), conforme Tabela 2.

Notou-se, nos dias D1 e D4, que o consumo de macronutrientes pelos pacientes é reduzido apesar de ter a menor variação nas quantidades tanto para proteínas quanto para carboidratos e lipídios. O consumo alcança o ápice no D3, no qual a quantidade consumida de macronutrientes é maior, embora seja observada uma variação elevada em relação ao D1, conforme Tabela 3.

$\mathrm{Na}$ Figura 1, verifica-se que o ano de acompanhamento com a maior diferença significativamente de calorias prescritas em relação a infundidas $(\mathrm{p}<0,001)$ foi 2010 com média de $-528.23 \mathrm{kcal}$. Já o ano que apresentou significativamente a menor diferença de calorias prescritas e infundidas foi $2011 \mathrm{com}$ média de $-481.27 \mathrm{kcal}$ $(\mathrm{p}<0,001)$.

Observou-se que mais de dois terços dos pacientes avaliados (62,5\%) sequer receberam $70 \%$ da TNE prescrita. Deste total, prevaleceram homens $(54,7 \%)$, havendo para essa associação estatisticamente significativa $\mathrm{p}=0,042$. A faixa etária mais frequente entre os pacientes de baixa infusão ( $<70 \%$ do volume prescrito) foi a de 38 a 54 anos (37,3\%); para os pacientes de baixa infusáo de 
Tabela 2. Comparação dos volumes prescrito e infundido e sua diferença nos dias de acompanhamento nutricionais em pacientes oncológicos sob terapia enteral. São Luís, Maranhão, Brasil

\begin{tabular}{|c|c|c|c|c|c|}
\hline \multirow[b]{2}{*}{$\begin{array}{c}\text { Dias } \\
\text { avaliados* }\end{array}$} & \multicolumn{2}{|c|}{ Volume de nutrição enteral (ml) } & \multirow{2}{*}{$\begin{array}{c}\text { Diferença de } \\
\text { volumes (ml) } \\
\text { Média } \pm \text { Desvio- } \\
\text {-padrão }\end{array}$} & \multicolumn{2}{|c|}{ Amplitude volume infundido (ml) } \\
\hline & Prescrito & Infundido & & Mínimo & Máximo \\
\hline D1 & $957,9 \pm 392,6$ & $480,9 \pm 244,9$ & $-477,0 \pm 409,9$ & 500 & 1.600 \\
\hline D2 & $964,9 \pm 392,4$ & $666,5 \pm 347,2$ & $-298,0 \pm 443,7$ & 600 & 1.500 \\
\hline D3 & $967,9 \pm 391,0$ & $981,7 \pm 513,8$ & $-261,4 \pm 382,1$ & 1.000 & 1.150 \\
\hline D4 & $966,3 \pm 393,0$ & $520,3 \pm 307,7$ & $-445,9 \pm 435,7$ & 1.000 & 1.500 \\
\hline
\end{tabular}

$\left({ }^{*}\right)$ Dia 1: Primeiro dia de acompanhamento; Dias 2 e 3: Sorteio entre os dias intermediários de acompanhamento nutricional; Dia 4: Último de acompanhamento.

Tabela 3. Consumo de macronutrientes diário de pacientes oncológicos em terapia enteral. São Luís, Maranhão, Brasil

\begin{tabular}{cccc}
\hline $\begin{array}{c}\text { Dias } \\
\text { avaliados* }\end{array}$ & $\begin{array}{c}\text { Proteína } \\
\text { (g/dia) }\end{array}$ & $\begin{array}{c}\text { Carboidrato } \\
\text { (g/dia) }\end{array}$ & $\begin{array}{c}\text { Lipídio } \\
\text { (g/dia) }\end{array}$ \\
\hline D1 & $24,7 \pm 15,7$ & $74,2 \pm 41,2$ & $24,1 \pm 16,7$ \\
D2 & $34,0 \pm 21,4$ & $111,8 \pm 59,8$ & $32,4 \pm 22,7$ \\
D3 & $38,1 \pm 22,2$ & $112,2 \pm 58,4$ & $37,4 \pm 23,6$ \\
D4 & $27,8 \pm 18,1$ & $81,4 \pm 52,2$ & $26,8 \pm 19,3$ \\
\hline
\end{tabular}

(*) Dia 1: Primeiro dia de acompanhamento; Dias 2 e 3: Sorteio entre os dias intermediários de acompanhamento nutricional; Dia 4: Último de acompanhamento.

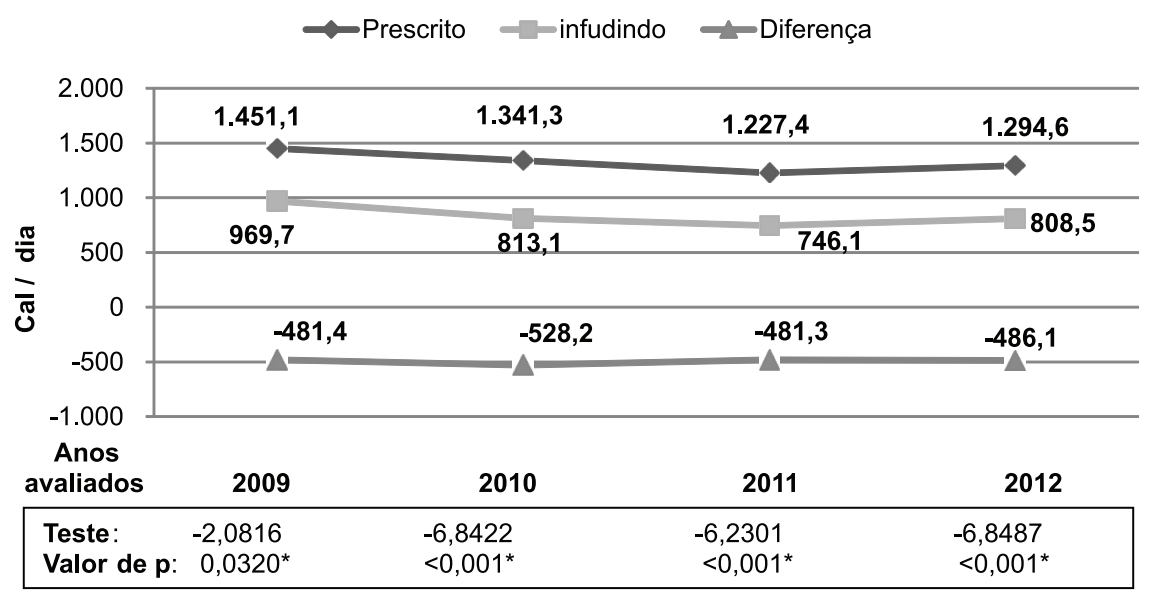

$\left.{ }^{*}\right)$ Associaçăo estatisticamente significativa.

Figura 1. Prescrição versus infusão e diferença média de energia na série temporal avaliada. São Luís, Maranhão, Brasil

TNE, prevaleceu a localização tumoral de cabeça e pescoço $(48,0 \%)$, conforme a Tabela 4 .

Os motivos mais frequentes relatados nas fichas para interrupção da dieta foram: atraso da dieta, dieta devolvida, problemas gastrointestinais, sonda obstruída, cirurgia e exames. Sendo que a dieta devolvida em virtude da recusa do paciente foi a intercorrência mais prevalente, $25,8 \%$ (31); e a menos frequente os problemas gastrointestinais $1,7 \%$ (dados não tabelados).

\section{DISCUSSÃO}

Neste estudo, prevaleceram homens adultos com tumores na região de cabeça e pescoço e de baixa infusão de TNE em relação ao prescrito. Ressalta-se que o fato de os pacientes não atingirem suas necessidades energéticas não é novo, estando relacionado ao favorecimento da desnutrição hospitalar, que já vem sendo estudada há décadas ${ }^{15}$.

No Inquérito brasileiro de avaliação nutricional hospitalar (IBRANUTRI), até $48,1 \%$ da população internada estudada encontrava-se desnutrida; destes, 
Tabela 4. Fatores associados ao indicador de qualidade em terapia nutricional (meta de infusão). São Luís, Maranhão, Brasil

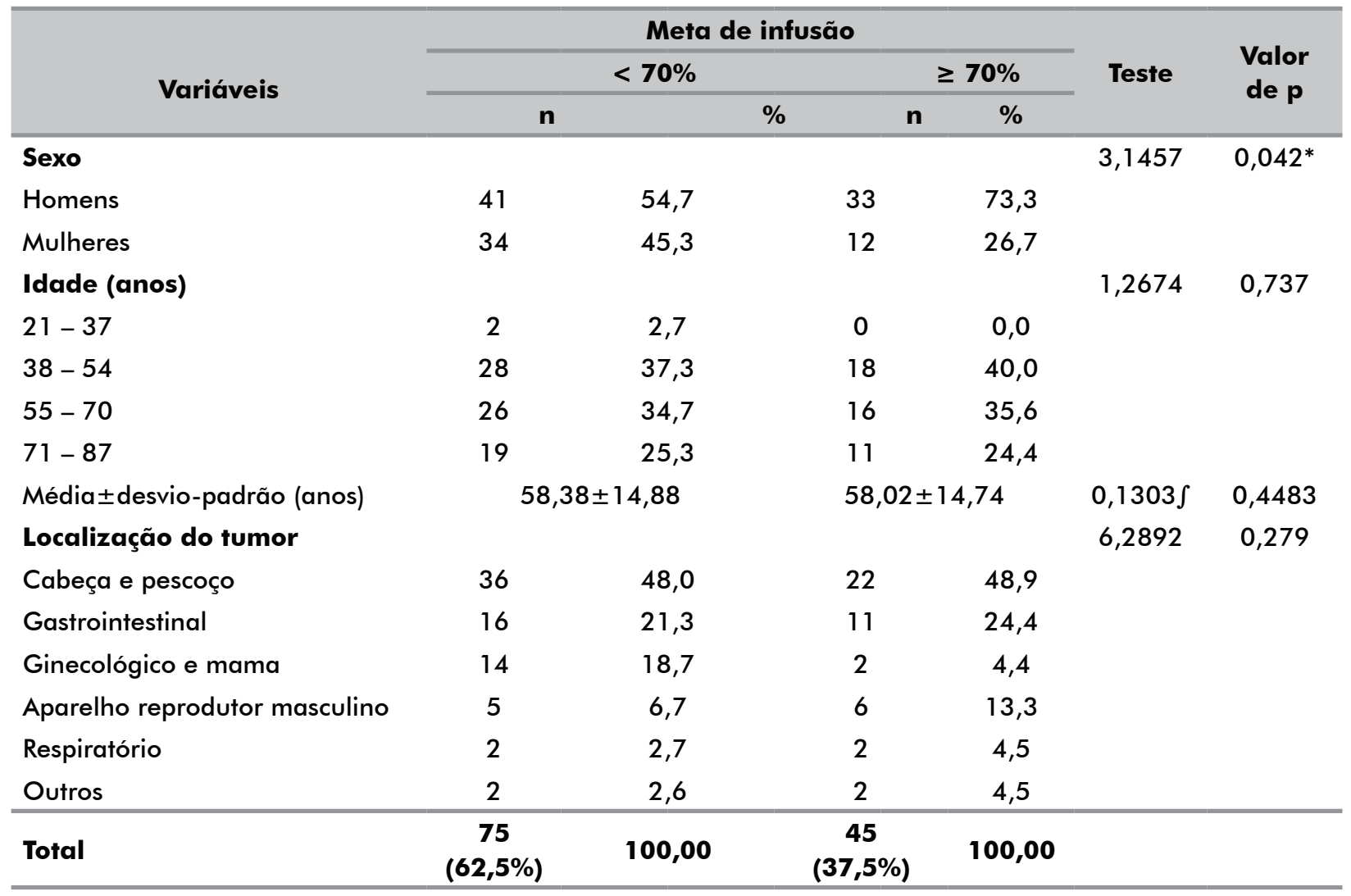

(*) Significância da associação entre sexo/gênero e meta de infusão ( $<70 \%$ ou >=70\%).

(J) Comparação da média de idade (variável contínua) entre a meta de infusão (<70\% e >=70\%).

$66,3 \%$ correspondiam a pacientes oncológicos ${ }^{15}$.

Mais tarde, quase duas décadas depois do IBRANUTRI, o Inquérito brasileiro de nutriçáo oncológica (IBNO) avaliou mais de quatro mil pacientes oncológicos pela avaliação subjetiva global produzida pelo próprio paciente (ASG-PPP), método padrão-ouro para essa população. Em comparação, o IBNO demostrou pouca alteração na taxa de prevalência de desnutrição nos pacientes avaliados, em que $45,1 \%$ apresentavam desnutriçáo moderada ou severa ${ }^{16}$.

Nota-se, em relação aos anos pesquisados, que o ano de 2010 apresentou a maior média de diferença entre o volume prescrito versus o infundido, podendo resultar em uma infusão significativamente inferior às necessidades metabólicas do paciente. Este mesmo ano apresentou a maior média de diferença entre a caloria prescrita e a de fato consumida pelos pacientes, podendo favorecer assim um balanço negativo da oferta energética.

Ressalta-se que o aporte calórico insuficiente pode contribuir para o agravamento do estado nutricional e consequentemente para o aumento do custo e do tempo de internação e morte.

No presente estudo, intercorrências como: recusa do paciente, pausas para realizaçáo de procedimentos cirúrgicos, realização de exames, atraso da dieta e problemas gastrointestinais foram os principais motivos citados para interrupção da infusão das dietas. Esses fatores já vêm sendo relatados como contribuintes de forma direta para essa diferença ${ }^{17}$.

Os principais motivos da meta da dieta prescrita não ser alcançada também são pontuados na literatura, por causa de procedimentos de enfermagem e de fisioterapia motora e respiratória, jejum para procedimento, problemas associados à sonda, à estase gástrica e à piora clínica. É possível que a quantidade diária da dieta administrada alcance em média 53\% das necessidades, sendo que mesmo as calorias prescritas ainda representavam $71 \%$ das necessidades reais ${ }^{12}$.

Procedimentos de fisioterapia e de enfermagem, embora possam causar atrasos na infusão da dieta, conforme pesquisa em pacientes hospitalizados em unidade de terapia intensiva, podem ter tempo recuperado durante as pausas noturnas, com uma maior flexibilidade no sistema de infusáo. Outras causas frequentes nas interrupçóes na infusão das dietas são o jejum para procedimentos (33\%), intolerâncias (29\%) e falhas na prescrição $(17 \%)^{18}$.

As principais intercorrências encontradas para tamanha diferença entre dieta prescrita e infundida se dão por procedimentos cirúrgicos, instabilidade clínica do paciente 
e remoção da sonda pelo paciente, seja ela voluntária ou acidental, sem a devida recolocação imediata ${ }^{19}$.

Há de se ressaltar que pacientes oncológicos, pela fisiopatologia da doença, tendem a ser menos tolerantes a elevados volumes de dieta. Pois, os fatores pró-anoréticos, interleucinas 1 e 6 , e o fator de necrose tumoral alfa podem reduzir a ingestáo calórica e, aliados a fatores produzidos pelo tumor (de mobilização de proteínas e de imobilização de lipídeos), alicerçam a cascata de perda de peso nesses pacientes ${ }^{20}$.

O presente trabalho possui como limitação a verificação de tais diferenças entre metas nutricionais prescritas versus infundidas nos dias iniciais (D1) e finais (D4); contudo, a verificaçáo ao longo dos dias D2 e D3 permite ao leitor uma noçẫo da flutuação dessa prescriçấo durante a utilização de TNE por esses pacientes. Além disso, a não avaliação das necessidades nutricionais dos pacientes também configurou uma limitação nesta pesquisa.

Assim, neste estudo, houve uma discrepância expressiva na infusão calórica e proteica com a prescrição dietética. Menos de um quarto dos avaliados alcançou a meta de infusão da dieta proposta pelo indicador de qualidade em "terapia nutricional volume infundido versus volume prescrito". Desta forma, são necessários novos estudos que se dediquem a avaliar as diferenças entre as necessidades de calorias e proteínas prescritas e infundidas para o adequado aporte nutricional dos pacientes oncológicos. Ressalta-se ainda a importância de se definir metodologias precisas para tal.

\section{CONCLUSÃO}

A terapia nutricional inclui uma série de medidas que devem ser instituídas o mais precocemente possível. A presença de uma equipe multiprofissional em terapia nutricional, a realização de treinamentos específicos e a aplicação de um protocolo de ação, a fim de proporcionar melhorias no estado nutricional dos pacientes e, consequentemente, nas funçôes imunológica e fisiológica, e mesmo na redução de risco e intercorrências relacionadas à dieta, podem configurar estratégias nutricionais promissoras.

\section{AGRADECIMENTOS}

Ao Hospital do Câncer Aldenora Bello.

\section{CONTRIBUIÇÕES}

Mikelly Joanny da Silva, Thalia de Jesus Souza da Silva, Jorge Antônio Pavão Cordeiro Sobrinho, Erika Ribeiro Garcia, Maria Tereza Silva de Medeiros, Thalita
Alburquerque Véras Câmara, Rosângela Maria Lopes de Sousa e Alexsandro Ferreira dos Santos contribuíram na concepção ou desenho do trabalho; aquisição, análise e interpretação dos dados da pesquisa; e na redação ou revisáo crítica com contribuição intelectual. Todos os autores aprovaram a versão final a ser publicada.

\section{DECLARAÇÃO DE CONFLITO DE INTERESSES}

Nada a declarar.

\section{FONTES DE FINANCIAMENTO}

Não há.

\section{REFERÊNCIAS}

1. Instituto Nacional de Câncer José Alencar Gomes da Silva. Estimativa 2020: incidência de câncer no Brasil. Rio de Janeiro: INCA; 2019.

2. Cattafesta M, Siqueira JH, Podestá OPG, et al. Consumo alimentar de pacientes com câncer de mama acompanhados em centro especializado em oncologia na Grande Vitória/ES-Brasil. Rev Bras Oncol Clín. 2014;10(38):124-31.

3. Ruivo EA, Fazeres FQ, Ventura J, et al. Impacto do suporte nutricional precoce na morbimortalidade em doentes submetidos a cirurgia de resseção por adenocarcinoma gástrico. Rev Port Cir. 2015;(34):27-36.

4. Cardoso DH, Muniz RM, Schwartz E, et al. Cuidados paliativos na assistência hospitalar: a vivência de uma equipe multiprofissional. Texto Contexto Enferm. 2013;22(4):1134-41. doi: https://doi.org/10.1590/ S0104-07072013000400032

5. Kliger G, Perman M, Echenique S, et al. Terapia nutricional en el paciente crítico. In: Prado RA, Márquez HA, Moya DA. Nutrición enteral y parenteral. 2. ed. New York: McGraw Hill; 2012. p. 315-332.

6. Diestel CF, Rodrigues MG, Pinto FM, et al. Terapia nutricional no paciente crítico. Rev Hosp Univ Pedro Ernesto. 2013;12(3):78-84. doi: https://doi. org/10.12957/rhupe.2013.7533

7. Moynihan T, Kelly DG, Fisch MJ. To feed or not to feed: is that the right question? J Clin Oncol. 2005;23(25):62569. doi: https://doi.org/10.1200/JCO.2005.04.019

8. Castro MG. A influência da introdução de um programa de educação médica em terapia nutricional no desfecho dos pacientes em uma unidade intensiva [tese na Internet]. Sáo Paulo, SP: Faculdade de Medicina da Universidade de São Paulo; 2012. 107 p. doi: https://doi. org/10.11606/T.5.2012.tde-26102012-114000

9. Ribeiro LMK, Oliveira Filho RS, Caruso L, et al. Adequacy of energy and protein balance of enteral nutrition in intensive care: what are the limiting factors? 
Rev Bras Ter Intensiva. 2014;26(2):155-62. doi: https:// doi.org/10.5935/0103-507X.20140023

10. Ferreira IKC. Terapia nutricional em Unidade de Terapia Intensiva. Rev Bras Ter Intensiva. 2007;19(1):90-7. doi: https://doi.org/10.1590/S0103-507X2007000100012

11. Gambato J, Boscaini C. Adequação da prescrição dietética e sua associação com intercorrências em pacientes em uso de terapia nutricional enteral. Rev Bras Nutr Clin. 2015;30(4):338-43.

12. Stefanello MD, Poll FA. Nutritional status and enteral diet prescribed and received by patients of an intensive care unit. ABCS Health Sci. 2014;39(2). doi: https:// doi.org/10.7322/abcshs.v39i2.625

13. Cervo AS, Magnago TSBS, Carollo JB, et al. Eventos adversos relacionados ao uso de terapia nutricional enteral. Rev Gaúcha Enferm. 2014;35(2):53-9. doi: https://doi.org/10.1590/1983-1447.2014.02.42396

14. Waitzberg DL, Enck CR, Miyahira NS, et al. Projeto diretrizes: terapia nutricional: indicadores de qualidade. Associação Médica Brasileira, Conselho Federal de Medicina; 2011 ago 25.

15. Waitzberg DL, Caiaffa WT, Correia MI. Hospital malnutrition: the Brazilian national survey (IBRANUTRI): a study of 4000 patients. Nutrition. 2001;17(7-8):573-80. doi: https://doi.org/10.1016/ S0899-9007(01)00573-1

16. Instituto Nacional de Câncer José Alencar Gomes da Silva. Inquérito brasileiro de nutrição oncológica. Rio de Janeiro: INCA; 2013.

17. Campos DJ, Silva AFF, Souza MH, et al. Otimizaçáo do fornecimento calórico protéico na terapia intensiva nutricional enteral em unidade de terapia intensiva com o uso de protocolo. Rev Bras Nutr Clin. 2006;21(1):2-5.

18. Cartolano FC, Caruso L, Soriano FG. Terapia nutricional enteral: aplicação de indicadores de qualidade. Rev Bras Ter Intensiva. 2009;21(4):376-83. doi: https://doi. org/10.1590/S0103-507X2009000400007

19. Isidro MF, Lima DSC. Adequação calórico-proteica da terapia nutricional enteral em pacientes cirúrgicos. Rev Assoc Med Bras. 2012;58(5):580-6. doi: 10.1590/S0104https://doi.org/42302012000500016

20. Santos AF, Rabelo Junior AA, Campos FLB, et al. Scored patient-generated subjective global assessment: length of hospital stay and mortality in cancer patients. Rev Nutr. 2017;30(5):545-53. doi: https://doi.org/10.1590/167898652017000500001 